\title{
Tele-Dentistry Rising: The Apps of Pediatric Dentistry: An Up-To- Date Review
}

\author{
Karataban PK* \\ Assist.Prof.Dr., Bahcesehir University School of Dental Medicine, Turkey \\ Faculty of Dentistry, Prof.Dr. BAU International University, Georgia
}

*Corresponding author: Dr. Pinar Kiymet Karataban, DDS PhD, Assistant Professor, Bahcesehir University School of Dental Medicine; Professor, Faculty of Dentistry, Bau International University Dentistry Faculty, Batumi, Georgia, Email: pkarataban@hotmail.com; pinarkiymet.karataban@dent. bau.edu.tr; pinar.kiymet.karataban@bauinternational.edu.ge

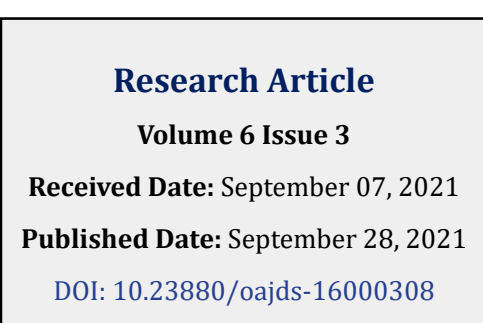

DOI: $10.23880 /$ oajds-16000308

\section{Abstract}

The pandemic changed the landscape of the accessibility to healthcare in an unexpected way. The instant disconnection with healthcare professionals including dental professionals thrusted tele-medicine and tele-dentistry into the spotlight. In pediatric dentistry, there are applications developed for both patients and dentists. This article aims to provide an overview of pediatric dentistry-related applications available on the Apple App Store and Google Play Store that can be useful for children, families and pediatric dentists. iPhone XS was used to access the Apple App Store, and Samsung Galaxy Note 20 devices was used to access the applications in the Google Play Store. The keywords; pediatric dentistry, teeth, brushing teeth, toothbrush timer, pediatric dental care, dentist games, dental pain, dental trauma were selected and searched in Google Play Store and Apple App Store. In total there were 313 applications related to pediatric dentistry which were classified as "Applications for Pediatric Dentists" and "Applications for Pediatric Patients and Their Families".

Keywords: Apps; Pediatric Dentistry; Tele-Dentistry; Tele-Medicine; Toothbrush Game; Covid-19 Pandemic

\section{Introduction}

Since the beginning of 21st century progress in the digitalization technology is undeniable. With the impact of Covid-19 pandemic and the sudden disconnection of the patients with the healthcare professionals, changed the landscape of the accessibility to healthcare and thrusted telemedicine and tele-dentistry into the spotlight.

Technology now constitutes a large part of our lives in both personal and professional fields. Especially in recent years, there has been an extraordinary increase in the adoption of new technological developments that change our lives. Along with technology, the development of smartphones is also on the agenda. Smartphones, besides receiving of phone calls and messages use a technology that allows routine access to limitless knowledge and information via access to the internet [1]. A smartphone is defined as a mobile device that displays advanced functions beyond a traditional mobile phone. Smartphones also can take photos, record and play videos, access e-mails, and browse the web [2].

With the development of operating systems, today, smartphones function like computers with their designs, and their ability to develop and use applications [3]. When analyzed the phone usage statistics in June 2021, 3.8 billion people in the world use smartphones which constitutes $48.33 \%$ of the world's population. The number of people with smart and feature phones is 4.88 billion, which corresponds to $62.07 \%$ of the world's population (Figure 1), [4]. 


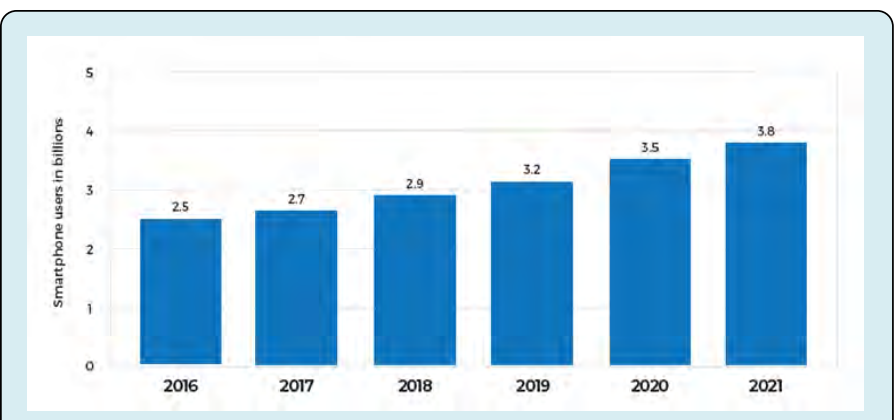

Figure 1: Number of smartphone users, worldwide iPhone data from 2016-2021 in billions [4].

With the use of smartphones, the use of mobile applications is also increasing. Mobile applications, commonly known as "apps", are small, functional, stand-alone software units designed to run through the information operating system of smartphones [5]. An application is a small special program that is usually downloaded to a mobile device, and a collection of applications can be accessed in Appstore for iPhone İOS System, and Google Play for Android System smartphones. All applications can be downloaded from this collection of applications $[1,6]$. Modern smartphones; iPhone and Android-based phones, can run third-party applications that provide unlimited functionality [2].

The first market entry of IOS App Store started with only 500 applications in 2008, comparing the data for 2021, approximately 1.85 million different applications are available in the App Store. In the Google Play Store for Android users, this number is currently 2.56 million [7].

\section{The Use of Apps in Healthcare and Dentistry}

Mobile applications also spread in fields such as medicine, public health, and dentistry [8]. These applications eliminate time constraints by providing health services to people at any time of the day. Location at the desired location allows people to access information without geographical barriers $[9,10]$. Since January 2020 , the download rate of applications related to the medical field has reached $65 \%$ [7]. This rise may also be related to the lockdowns and restrictions in the Covid-19 Pandemic period. Besides, during the pandemic period the need to access to healthcare professionals for the non-emergency conditions, might have led the development of more telemedicine apps.

According to Statista data for 2021, the number of current health and medical applications is 53,054 in the Google Play Store and 53,9792 in the IOS Apple Store [11,12]. With the technological development of smartphones, applications have also evolved and currently there are applications both clinicians and students can benefit from in areas such as smoking cessation [13], pain management [14], orthopedic surgery [15], ophthalmology [16], and radiology [17]. The field of dentistry is also affected by these developments, and the number of applications related to the field is increasing day by day. Bohn, et al. evaluated the approaches of patients to dental applications, and they found that patients liked the applications and found them educational and useful; and that the applications improved the patients' communication with the dentist [18].

In pediatric dentistry, there are applications developed for both patients and dentists. There are applications in pediatric dentistry which provide opportunity for pediatric dentists to integrate technology into their clinical practices, to instill oral hygiene habits for pediatric patients. For parents there are game like apps which help to provide oral hygiene motivation for their children, besides there are medicinal apps which help them to cope with the dental emergency cases such as dental trauma. Besides many advantages of the useful applications, the presence of incorrect or incomplete information in some applications may cause disadvantages such as delaying dentist appointments by adhering to the information in the applications. The advantages and disadvantages of applications related to pediatric dentistry are listed in Table 1.

\begin{tabular}{|c|c|}
\hline Advantages & Disadvantages \\
\hline Easy and fast access to information & $\begin{array}{l}\text { The risk of inaccurate and incomplete information in } \\
\text { applications }\end{array}$ \\
\hline Providing oral hygiene education and motivation to family and child & $\begin{array}{l}\text { Disruption of dentist appointments by adhering to } \\
\text { the information in the applications. }\end{array}$ \\
\hline \multicolumn{2}{|l|}{ Preparing the child for dental procedures } \\
\hline \multicolumn{2}{|l|}{ Preventing dental phobia in children } \\
\hline \multicolumn{2}{|l|}{ Enabling family to cope with emergencies such as dental trauma } \\
\hline \multicolumn{2}{|l|}{ Dentists' ability to integrate technology and clinical practices } \\
\hline Ease of access for dentists to new, up-to-date information. & \\
\hline
\end{tabular}

Table 1: Advantages and Disadvantages of Applications in Pediatric Dentistry. 


\section{Open Access Journal of Dental Sciences}

\section{Methods}

This article is relevant to pediatric dentistry apps available on the Apple App Store (Apple Inc., Cupertino, CA, USA) and the Google Play Store (Google Inc., Mountain View, CA, USA), to both children and family.

On 13 June 2021, 2 smartphones were used to access 2 different stores of applications. Iphone XS Max (Apple Inc., Cupertino, CA, USA) device with IOS 14.6 software installed was used to access the Apple App Store. To access the applications in the Google Play Store, the Samsung Galaxy Note 20 (Samsung Telecommunications, Suwon, Korea) device with the R Android 11 software was used. Keywords selected searched in Google Play Store and Apple App Store were as follows; pediatric dentistry, teeth, brushing teeth, toothbrush timer, pediatric dental care, dentist games, dental pain, dental trauma. The research included paid and free applications related to pediatric dentistry in all languages. Applications in different languages were analyzed by translating with Google Translate (Google Inc.). All information collected for each application, including the price details of the application and about the developer was recorded and included in the study. All other dentistry and medical health applications that are not related to pediatric dentistry were excluded from the study.

\section{Results}

All applications related to pediatric dentistry were categorized into 4 main groups according to the application store type and application's interest to patients or pediatric dentists. Applications that interest children and families according to the detailed contents were classified into 4 subcategories as games, brush timers and simulators, general information for families, and dental emergencies. Applications for pediatric dentists are classified into 4 subcategories: practice management and diagnosis aid, publications/journals/news, dental emergency, and finally pediatric dental drugs.

The applications were classified as "Applications for Pediatric Dentists" and "Applications for Pediatric Patients and Their Families." While there are 20 applications in the App Store for pediatric dentists, there were 38 applications in the Google Play Store. While the number of applications for pediatric patients and their families was 151 in the App Store, it was 162 in the Google Play Store. As a result, it was determined that there were a total of 313 applications related to pediatric dentistry (Table 2).

\begin{tabular}{|c|c|c|}
\hline & Applications for Pediatric Dentists & 7 \\
\hline & Practice Management/ Aiding Diagnosis & 5 \\
\hline & Publications/Journals/News & 1 \\
\hline & Dental Emergency & 7 \\
\hline & Pediatric Dental Drugs Management & 82 \\
\hline & Gpplications for Pediatric Patients and Their Families & 9 \\
\hline & General Information for Families & 59 \\
\hline & Brush Timers and Simulations for Children & 1 \\
\hline & Dental Emergency & 151 \\
\hline & TOTAL & 14 \\
\hline & Applications for Pediatric Dentists & 8 \\
\hline & Practice Management/ Aiding Diagnosis & 6 \\
\hline & Publications/Journals/News & 10 \\
\hline & Dental Emergency & \\
\hline & Pediatric Dental Drugs Management & 96 \\
\hline & Gplications for Pediatric Patients and Their Families & 14 \\
\hline & Games & 46 \\
\hline & Brush Timers and Simulations for Children & 6 \\
\hline & Totoren & 162 \\
\hline
\end{tabular}

Table 2: Available Applications for IOS and Android. 


\section{Applications for Pediatric Dentists}

As a result of screening applications for pediatric dentists in both Appstore and google play a total of 58 apps were identified. Applications that generally help in clinical practice, apps that contribute to the diagnosis, treatment of diseases, and apps that enable the dental professional to follow innovations and developments in the field of pediatric dentistry. To better understand the content and spectrum of these applications, we categorized them under 4 categories as; "Practice Management/ Aiding Diagnosis", "Publications/ Journals/News", "Dental Emergency", and "Pediatric Dental Drugs Management".

There were a total of 21 applications for iOS and Android phones in the "Practice Management/Aiding Diagnosis" area. The Handbook of Pediatric Dentistry, developed by The American Academy of Pediatric Dentistry (AAPD), which is among the applications, is an application available in both the App Store and Google Play Store (Figure 2). It provides AAPD members to access information easily and quickly. In the content of the application, some sections can help pediatric dentists in their clinical practice and at the same time follow the innovations in this field. The most up-to-date approaches to diagnosis and treatment in pediatric dentistry can be accessed from the login, Search the Handbook, Browse the Handbook by Chapter, Member Website, Authors \& Intro, Oral Health Policies \& Recommendations options.

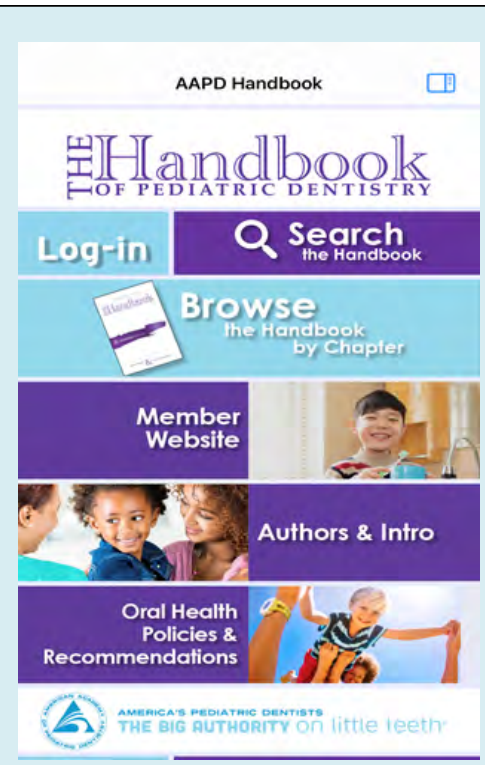

Figure 2: The Handbook of Pediatric Dentistry.

In total, there were 13 applications for IOS and Android phones in the "Publications/Journals/News" area. Journal Paediatric Dentistry, which is among the applications, is a journal application (Figure 3). It includes case studies, the most up-to-date studies, and publications, where developments in the field of pediatric dentistry are explained. It is an application available for both iOS and Android devices. Thus, pediatric dentists can access up-to-date information anytime, anywhere, thanks to this application regardless of place and time. Another feature of the application is that it sends notifications when new journals are published, allowing pediatric dentists to be informed instantly. In addition, it offers easy access to these notes by downloading the magazines, adding notes to important places, and making highlights.

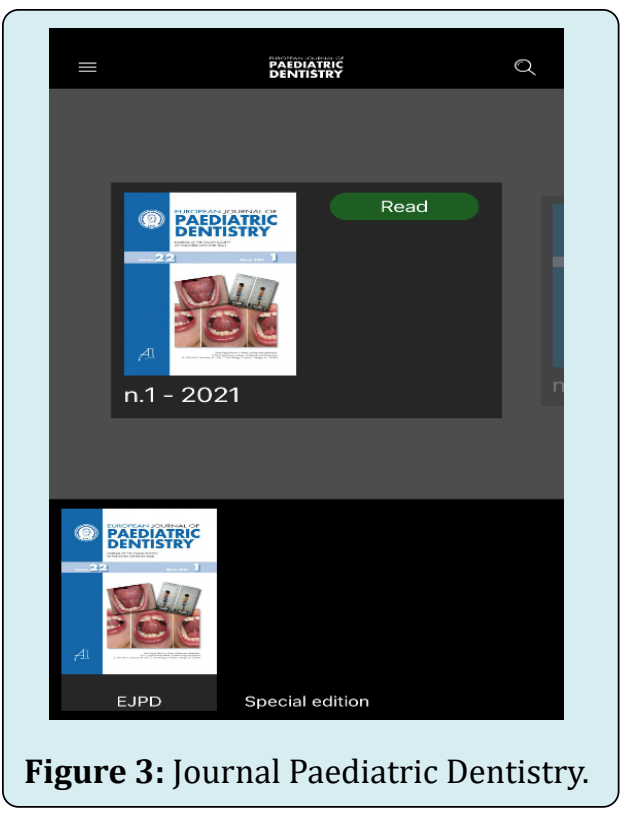

There are a total of 7 applications for IOS and Android phones in the "Dental Emergency" field. Applications in this area concern both pediatric dentists and families. The ToothSOS application is an application affiliated with the International Association of Dental Traumatology (Figure 4).

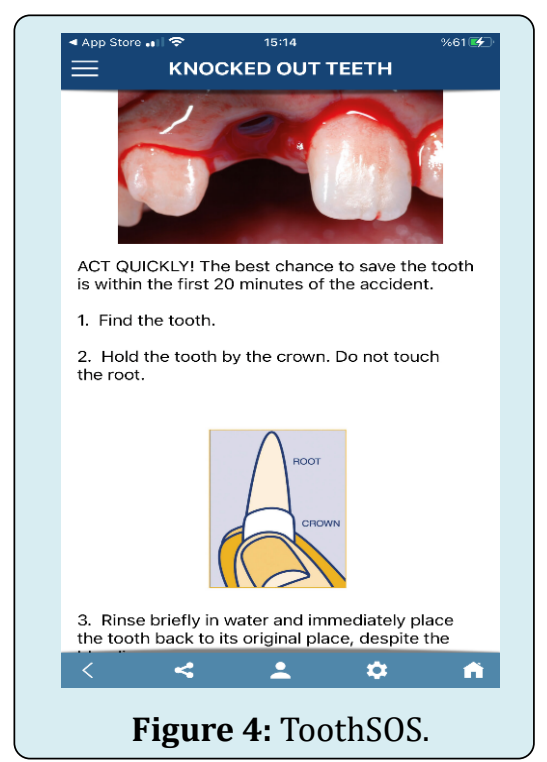


It is a downloadable application for iOS and Android phones. There are "I Have a Tooth Injury" and "How to Prevent Dental Injuries" options for families. After selecting the type of emergency dental trauma in the "I Have a Tooth Injury" option, the steps that the person should follow at that moment are explained in detail according to the trauma type. In the "How to Prevent Dental Injuries" option, people are informed about what needs to be considered to prevent trauma. The latest dental trauma guide is available for dentists, and emergency treatment options are offered to dentists in patients who come with trauma. In addition, continuing education on trauma is also available in this application.

In the last category of applications for pediatric dentists, there were a total of 17 applications for iOS and Android phones under the title of "Pediatric Dental Drugs Management". Pediatric Dental Dosing application is a downloadable application for both iOS and Android devices (Figure 5). It has very useful content that allows the pediatric dentist to adjust the dose according to the age and weight of the child and provide detailed information about the drug to be prescribed when the drug is to be prescribed for pediatric patients.

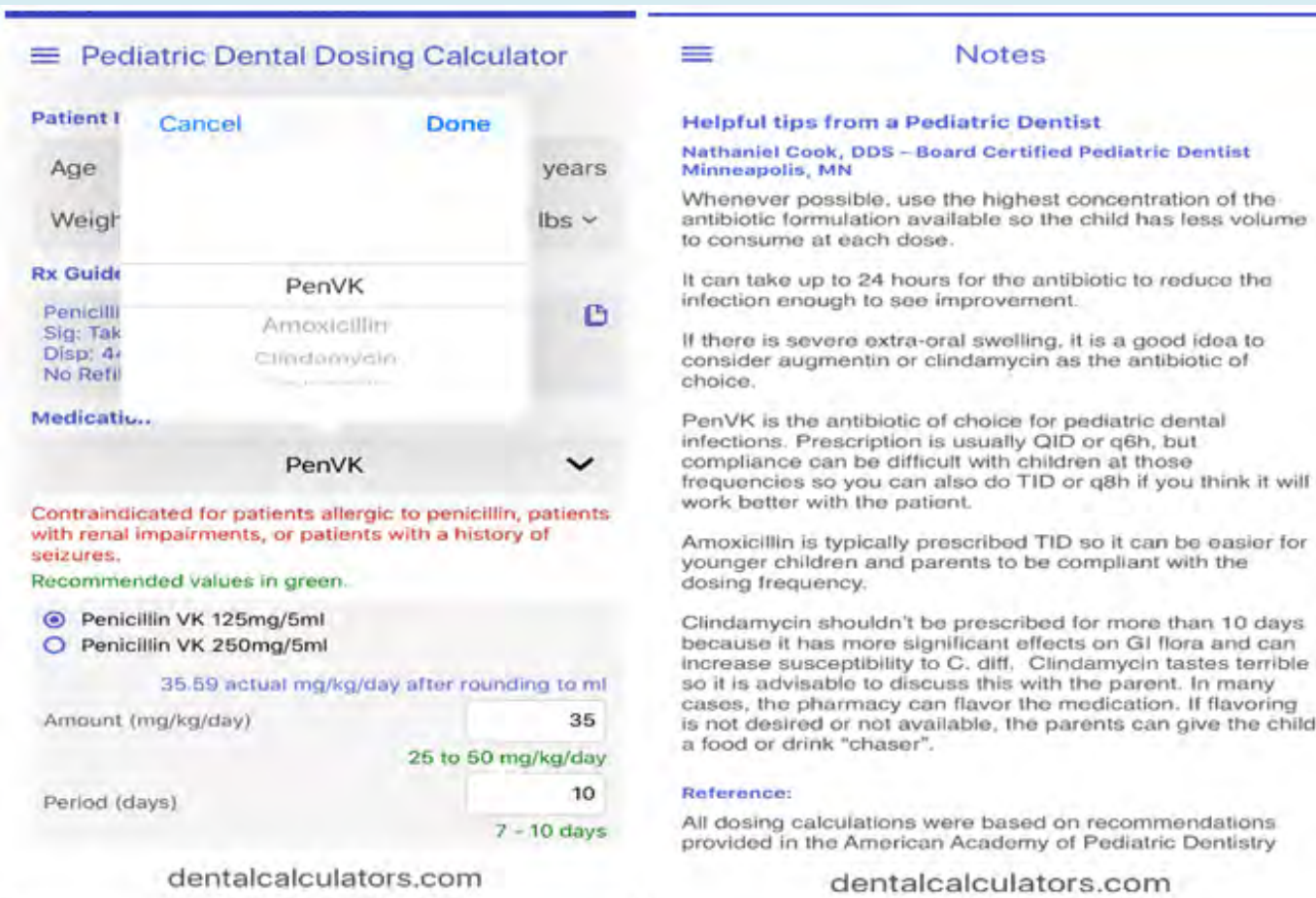

Figure 5: Pediatric Dental Dosing Calculator.

\section{Applications for Pediatric Patients and Their Families}

As a result of screening applications a total of 313 apps for pediatric patients and parents have been revealed. Tooth brushing simulations and timers that enable children to make tooth brushing habits more fun and games that help them overcome their fear of the dentist have been identified. For families, there are informative applications that enable their children to perform oral hygiene and dental care correctly and guide them and applications that explain what needs to be done in emergency dental traumas. To better understand the content and areas of these applications, we have categorized them under 4 categories as; "Games", "General
Information for Families", "Brush Timers and Simulations for Children", and "Dental Emergency".

There are a total of 178 applications for IOS and Android phones in the "Games" category. Dentist Care: The Teeth Game is available on the App Store and Google Play Store (Figure 6). With the simpler versions of the equipment used by pediatric dentists, cavities are cleaned and dental treatments of the characters in the application are carried out. In this way, this game provides a fun and simple rehearsal for children to understand that dental treatment is a good thing and how they will encounter the procedures when they go to the dentist by mastering these stages. 


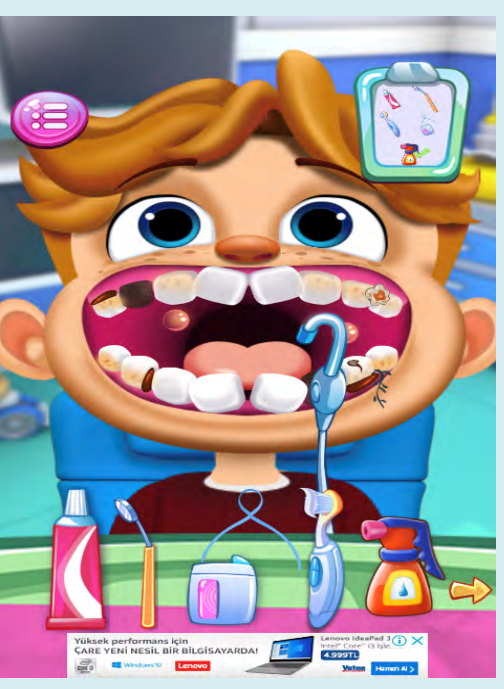

Figure 6: Dentist Care: The Teeth Game.

In the "General Informations for Families" category, there are a total of 23 applications in both the Google Play Store and the App Store. The Dental App is an application that provides families with the necessary information to maintain their children's dental health (Figure 7). It enlightens families in detail about the frequency of children's tooth brushing, the frequency of dental visits, and breastfeeding.

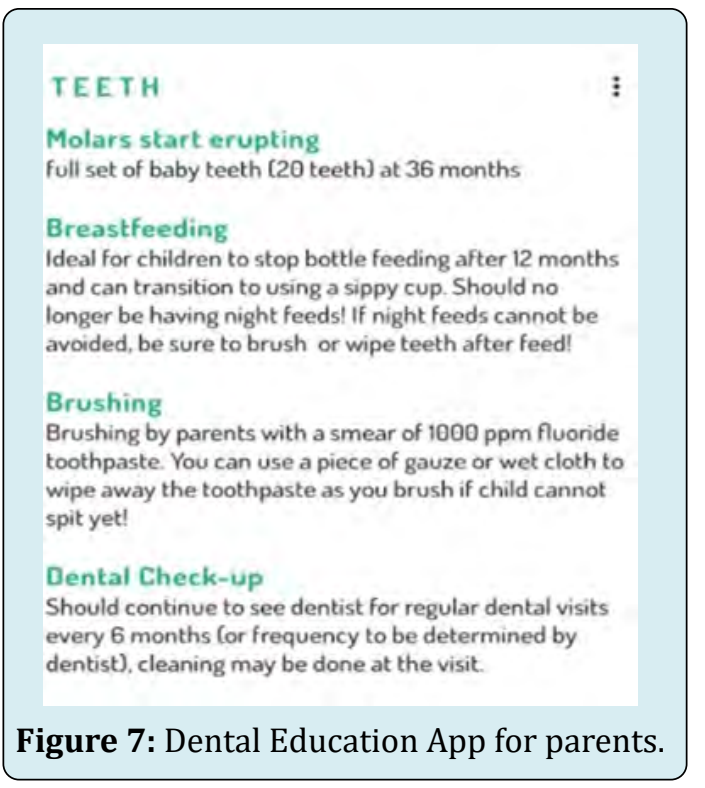

There are a total of 105 applications for iOS and Android devices in the "Brush Timers and Simulations for Children" section (Figure 8). The brushing Hero application is a simulation application that encourages children to brush their teeth. In the application, after the phone's camera is turned on, the child becomes a hero and as he brushes his teeth, the creatures are killed and gold is earned. This application, which allows the child to level up as they accumulate points and gold, makes brushing fun.

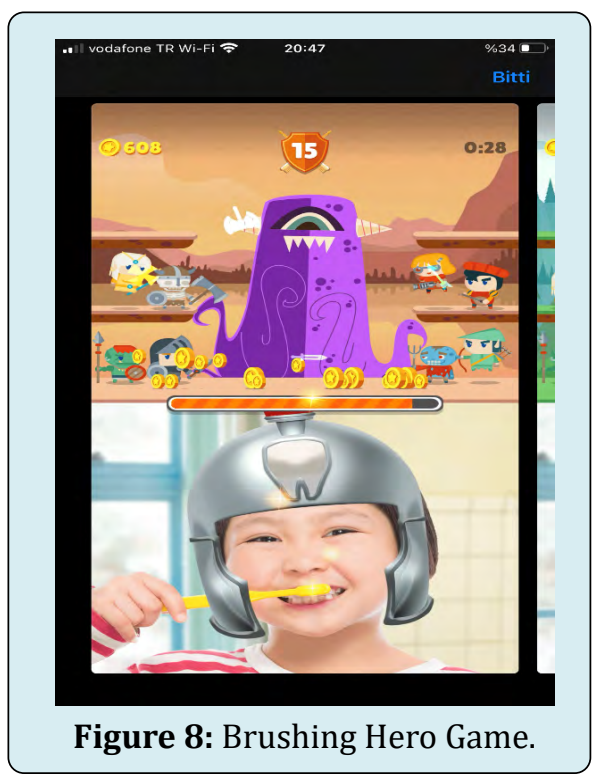

Under the last category, "Dental Emergency", there are a total of 7 applications for iOS and Android devices. The Dental Trauma application is available on the Google Play Store (Figure 9). In this application, what family should do in case of traumatic dental injuries, is explained in detail according to the type of trauma that occurs in the permanent or primary teeth of the children. This app works allowing the family to control the situation healthily until they go to the dentist.

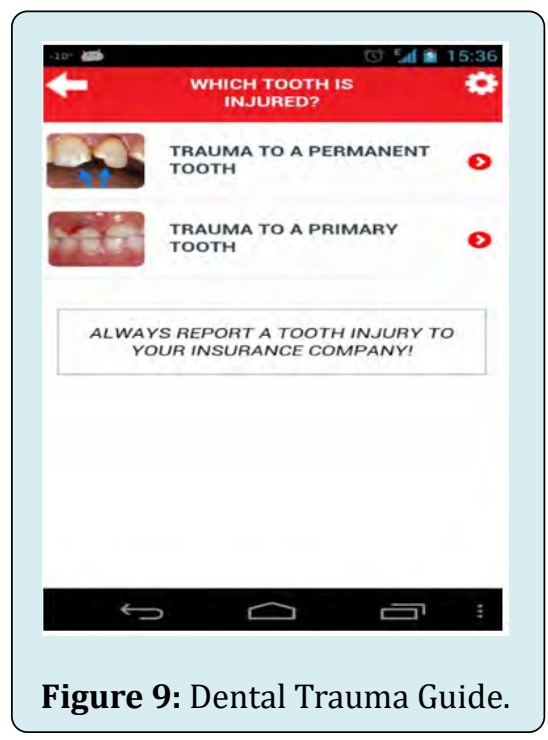

\section{Current Perspectives}

The technological development and innovations in the field of dentistry are now developing towards digitalization. The increase in development of applications, especially in 
the field of pediatric dentistry, is very beneficial for pediatric dentists, families and their children. While it is an important advantage for pediatric dentists to be able to follow information and innovations anywhere at any time, it is now important for families and children to increase awareness of such practices as oral hygiene and tooth brushing. We scanned the applications available in the Google Play Store and Apple App Store in this study. Of the 313 applications targeting parents and children related to pediatric dentistry, 162 belong to the Google Play Store and 151 to the Apple App Store.

When all the applications are analyzed it is seen that there are many applications in the categories of "Games" and "Brush Timers and Simulations for Children", especially in applications for pediatric patients and families. This shows that the importance given to children's dental health has increased by associating it with technology. There is a need to increase the number of applications in other categories, especially in applications for pediatric dentists, and in the category of "general information for families".

\section{Conclusion}

The number of health applications, especially in the field of pediatric dentistry, is increasing day by day. Although there are many advantages of applications in this field, it is also crucial to pay attention to the disadvantages. Most of the applications are not developed by a dental professional nor with the help of an association of pediatric dentistry. There are apps aiming the education of parents but the educational material is not sufficient. For this reason, it is necessary to make arrangements to prevent children and their families from accessing erroneous content in applications.

In addition, considering all these developments, families should not trust the effectiveness of the applications one hundred percent and not disrupt their children's dental examinations. Pediatric dentists should also use the applications they deem appropriate as a source of motivation for their families and children. They should warn the family that these applications are only motivating methods and should not interfere with regular examinations and treatments to pediatric dentists.

\section{Acknowledgement}

I would like to express my appreciation to Assoc. Prof.Dr. Delal Dara Kılınç and Dr. Irmak Erakman for their valuable suggestions during the planning and development of this research work.

\section{References}

1. Singh P (2013) Orthodontic apps for smartphones. J Orthod 40(3): 249-255.

2. (2021) Smartphone.

3. Boulos MNK, Wheeler S, Tavares C, Jones R (2011) How smartphones are changing the face of mobile and participatory healthcare: an overview, with an example from eCAALYX. BioMed Eng OnLine 10: 24.

4. (2021) Cell phone Research \& Guides.

5. Wallace S, Clark M, White J (2012) 'It's on my iPhone': attitudes to the use of mobile computing devices in medical education, a mixed-methods study. BMJ Open 2(4): e001099.

6. Mamillapalli PK, Neela PK, Sesham VM (2012) Model analysis on a smartphone. JCO 46(06): 256-258.

7. https://www.businessofapps.com/data/app-statistics

8. Kay M, Santos J, Takane M (2011) mHealth: New horizons for health through mobile technologies. WHO.

9. Akter S, Ray P (2010) mHealth-an Ultimate Platform to Serve the Unserved. Yearbook of medical informatics pp: 94-100.

10. Thakkar J, Barry T, Thiagalingam A, Redfern J, McEwan AL, et al. (2016) Design Considerations in Development of a Mobile Health Intervention Program: The TEXT ME and TEXTMEDS Experience. JMIR MHealth UHealth, 4(4): e127.

11. https://www.statista.com/statistics/779919/healthapps-available-google-play-worldwide/

12. https://www.statista.com/statistics/779910/healthapps-available-ios-worldwide/

13. Abroms LC, Padmanabhan N, Thaweethai L, Phillips T (2011) iPhone apps for smoking cessation: a content analysis. Am J Prev Med 40(3): 279-285.

14. Rosser BA, Eccleston C (2011) Smartphone applications for pain management. J Telemed Telecare 17(6): 308312.

15. Franko OI (2011) Smartphone apps for orthopaedic surgeons. Clin Orthopaed Relat Res 469(7): 2042-2048.

16. Hassani TJR, El Sanharawi M, Dupont Monod S, Baudouin C (2013) Smartphones in ophthalmology. Journal francais d'ophtalmologie 36(6): 499-525. 


\section{Open Access Journal of Dental Sciences}

17. Rodrigues MA, Visvanathan A, Murchison JT, Brady RR (2013) Radiology smartphone applications; current provision and cautions. Insights Imaging 4(5): 555-562.

18. Bohn CE, McQuistan MR, McKernan SC, Askelson NM
(2018) Preferences Related to the Use of Mobile Apps as Dental Patient Educational Aids: A Pilot Study. J Prosthodont 27(4): 329-334.

(a) (1) 